\title{
Review of: "Research On Medical Insurance Policies Against Serious Illness Among Urban And Rural Residents In Shanghai Based On Micro-Simulation"
}

\section{Zhang Xinjie}

Potential competing interests: The author(s) declared that no potential competing interests exist.

This study actually discusses the sustainability of Shanghai's serious illness protection policy, which is of great significance in itself. However, the simulation method adopted in this study is too simple. The operation of medical insurance fund will be affected by many factors, such as the outbreak of COVID-19 in 2020. Therefore, more accurate actuarial model is needed for calculation. In addition, although the medical insurance fund is pay-as-you-go, it still requires to consider the medium- and long-term balance of the fund in the policy making process. It can be seen that the forecast period of this paper is also too short, and the long-term effect of the policy cannot be fully foreseen. 